\title{
TIDAL TENSOR AND THE EMISSION AND ABSORPTION OF GRAVITATIONAL RADIATION
}

\author{
TERRENCE J. SEJNOWSK I
}

University of California at Santa Barbara, Calif., U.S.A.

(Presented by J. B. Hartle)

\begin{abstract}
The absorption and emission of gravitational radiation can be calculated in the long wavelength limit by use of the tidal tensor, defined as the gradient of the gravitational pseudoforce,

$$
\left(\begin{array}{c}
\text { local 'force' } \\
\text { on } T_{a b}
\end{array}\right) \equiv\left(\sqrt{-g} T^{a b}\right)_{, b}=f^{a} ;
$$

thus

$$
\text { (tidal sensor) }{ }_{d}^{a} \eta^{d} \equiv f^{a}{ }_{, d} \eta^{d}=\frac{2}{3} R^{a}{ }_{(b c) d} T^{b c} \eta^{d}+O\left(\eta^{2}\right),
$$

where $\eta^{b}$ is orthogonal to the 4-velocity $u^{a}$, and normal coordinates are understood.

The exchange of energy and momentum between an extended body and the gravitational field is governed by appropriate integrals of the tidal tensor over space and time.

The tidal tensor is the trace $T^{a}{ }_{b d}^{b}$ of the Bel-Robinson tensor $T_{a b c d}$. In emty space this trace is zero and the tidal tensor vanishes; there is no local exchange of energy.
\end{abstract}

